\title{
Processing and properties of Titanium alloy based materials with tailored porosity and composition
}

\author{
Jose Luis Cabezas-Villa ${ }^{1,2}$, Luis Olmos ${ }^{1}$, Jose Lemus-Ruiz ${ }^{1}$, Didier Bouvard ${ }^{2, *}$, Jorge Chavez ${ }^{3}$, Omar Jimenez ${ }^{3}$, and Victor \\ Manuel Solorio 4 \\ ${ }^{1}$ Universidad Michoacana de San Nicolás de Hidalgo, IIMM and INICIT, Fco. J. Mujica S/N, Morelia, Michoacán, C.P. 58060, México \\ ${ }^{2}$ Univ. Grenoble Alpes, CNRS, SIMAP, 38000 Grenoble, France \\ ${ }^{3}$ Universidad de Guadalajara, Departamento de Ingeniería de Proyectos, José Guadalupe Zuno \# 48, Jalisco, C.P. 45100, México \\ ${ }^{4}$ Tecnológico Nacional de México, Posgrado de Ciencias en Metalurgia ITM, Av. Tecnológico \# 1500, Morelia, C.P. 58120, México
}

\begin{abstract}
This paper deals with powder processing of Ti6Al4V titanium alloy based materials with tailored porosity and composition. Ti6Al4V powder was mixed either with salt particles acting as space holder, so as to provide two-scale porosity, or with hard TiN particles that significantly modified the microstructure of the material and increased its hardness. Finally an original three-layer component was produced. Sample microstructure was observed by SEM and micro-tomography with special interest in pore size and shape, inclusion distribution and connectivity. Compression tests provided elastic modulus and yield stress as functions of density. These materials are representative of bone implants subjected to complex biological and mechanical conditions. These results thus open avenues for processing personalized implants by powder metallurgy.
\end{abstract}

\section{Introduction}

Powder metallurgy is the ideal route to produce parts with complex composition, microstructure and geometry. The critical step is sintering, during which the final properties and dimensions are obtained. Nevertheless, the possibility of fabricating components including several regions with various characteristics (dense, porous, composite) has hardly been considered. This is because of the difficulty of cosintering materials with different densification rates without generating cracks at the interfaces. Yet, the interest of such "architectured" materials with multifunctional properties is increasing in various fields, in particular in biomedical engineering for bone replacement. Titanium alloys have been proposed as an alternative to enhance the lifespan of orthopedic implants because of their excellent combination of mechanical and corrosive properties. In the past two decades, many works have been devoted to defining the most appropriate method to fabricate dense and porous Ti6Al4V parts by powder metallurgy $[1,2]$. In this work, we investigated the sintering of various systems based on biocompatible Ti6Al4V titanium alloy with the objective of producing architectured layered materials with suitable features allowing for their use as advanced bone implants. First, materials with two scale porosity have been fabricated from the mixture of the powder with salt particles acting as space holders. Next, the sintering of Ti6Al4V powder mixed with TiN particles has been investigated. Finally, we have been able to produce a three-layer component comprising a close-to-dense layer, a highly porous layer and a reinforced layer. The microstructure of every component has carefully been characterized by SEM and microtomography and its mechanical properties have been evaluated by compression and microhardness tests.

\section{Materials and methods}

A prealloyed powder of Ti-6Al-4V with spherical particles smaller than $20 \mu \mathrm{m}$, produced by Raymor, Quebec Canada, was used as the matrix material. Those powders were mixed (1) with particles of ammonium bicarbonate ((NH4)HCO3) with irregular shape and size distribution between 100 and $500 \mu \mathrm{m}$, provided by Alfa Aesar (USA), for acting as space holder in porous samples, (2) with angular TiN particles with particle size below $45 \mu \mathrm{m}$, supplied by AEE Inc., to produce composites. Powders were mixed in a Turbula during 30 minutes. Then, $10 \mathrm{wt} \%$ of polyvinyl alcohol (PVA) was added 
as a binder. Next, the mixture was poured into an 8 $\mathrm{mm}$ diameter stainless steel die and pressed at 500 MPa using an Instron 1150 universal machine to obtain cylindrical compacts of $10 \mathrm{~mm}$ height. The fabrication of the three-layer sample includes the following steps: a layer of regular powders was poured into the die; then, the mixture to produce the porous layer was poured above; finally, the powder mixture to form the composite layer was poured onto the porous layer. This resulting sample was compacted at the same pressure.

In every case, space holder particles were removed at $150{ }^{\circ} \mathrm{C}$ during $6 \mathrm{~h}$ in air and the PVA was thermally extracted by a slow heating treatment at 5 ${ }^{\circ} \mathrm{C} / \mathrm{min}$ up to a temperature of $500^{\circ} \mathrm{C}$ with a dwell time of $40 \mathrm{~min}$ in argon. Afterwards, each compact was sintered in a vertical dilatometer Linseis $\mathrm{L} 75 \mathrm{~V}$ between 1150 and $1260{ }^{\circ} \mathrm{C}$ during $1 \mathrm{~h}$ in argon. The weight density of every sintered compact was calculated from mass and dimension measurements. The sintered compacts were cut and the surface was metallographically prepared with $\mathrm{SiC}$ papers for grinding and alumina powders (up to $50 \mathrm{~nm}$ particle size) for final polishing. The microstructure of sintered samples was observed with a Mira3 TESCAN Field-Emission Scanning Electron Microscope (FE-SEM). 3D imaging was performed by computed micro tomography (CMT) with a ZEISS Xradia 510 Versa microCT. The beam intensity was $120 \mathrm{kV}$, which was enough to pass through Ti6Al4V samples of $8 \mathrm{~mm}$ diameter. 1600 projections were taken around $360^{\circ}$ with a CCD camera of $2048 \times 2048$ pixels providing a pixel resolution of around $10 \mu \mathrm{m}$. Due this resolution matrix pores cannot be seen. The treatment of 3D images included filters to eliminate the noise and thresholding to distinguish between the solid and porous phases. Different morphological operations like opening, closing and erosion were achieved to improve the quality of the binary images. Finally, the pore volume fraction was calculated by dividing the number of voxels corresponding to the pore phase by the total number of voxels in the image. The pore size distribution was estimated by measuring the pore volume accessible to an octahedral structural element with increasing size, as proposed by various authors for interconnected porosity [3].

\section{Results and discussion}

\subsection{Porous compacts}

Fig. 1 shows the relative density of all sintered samples as a function of the volume fraction of salt. It also includes the density of the green compacts. It can be noticed that the initial relative density decreases almost linearly with increasing volume fraction of salt. It goes from 0.26 for the material with $50 \mathrm{vol} \%$ of salt to 0.7 for the material without salt. The final density also decreases with increasing fraction of salt and it increases with increasing sintering temperature. The minimum value, 0.28 , was obtained for the sample with $50 \mathrm{vol} \%$ of salt sintered at $1150^{\circ} \mathrm{C}$. Note that the samples obtained without salt have a density around 0.95 .

The pore size distribution estimated from 3D images (Fig. 3c and d) is shown in Fig .2 for three porous samples with 10,20 and 30 vol.\% of salt. The particle size distribution of the initial salt particles has been added as a reference value to analyze the pore size obtained after sintering. It can be seen that the pore size distribution is quite similar for all of porous samples, confirming the uniform distribution of salt particles inside of samples. However, it is notable that the $\mathrm{d}_{50}$ diameter of pores after sintering is about $35 \%$ smaller than the one of the salt particles. This difference should result from pore changes occurring during compression and sintering steps.

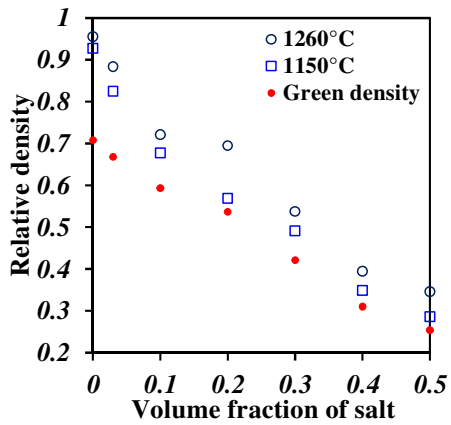

Fig. 1. Relative density as a function of the volume fraction of salt in the green state and after sintering

Fig.3 shows the microstructure of sintered samples observed by SEM and CMT. The matrix composed of Ti6Al4V particles is well sintered around the large pores, although, interparticle pores are visible. It appears that the matrix is less dense when the volume fraction of salt is higher (Fig. 3b) which suggests that sintering is perturbed by the presence of large pores. Fig. $3 \mathrm{~b}$ suggests that large pores are connected with each other through interparticle pores. These microstructures are different from those obtained by EBM because this technique melts the particles [4]. Figs $3 \mathrm{c}$ and $3 d$ show 3D renderings of the porosity inside the samples. Pseudo-colors are used to identify interconnected pores. It can be noticed that large pores are isolated from each other in the specimen with $10 \mathrm{vol} . \%$ of salt. Salt particles appear to be randomly distributed inside the sample because very 
few particle agglomerates are observed. On the other hand, with $30 \mathrm{vol} . \%$ of salt, the large pores are fully interconnected (Fig. 3d), which proves that these particles formed a continuous network inside the sample before being eliminated. Additional images showed that the transition between large pore separation and interconnection occurs at $20 \mathrm{vol} . \%$. Indeed, non-detected interparticle pores may connect large pores with each other even when 10 vol.\% of salt are used, as suggested by Fig. $3 b$.

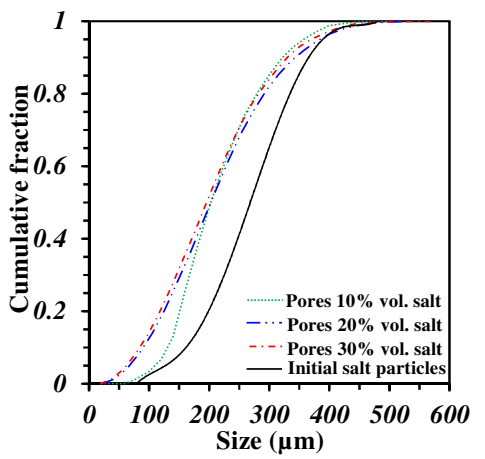

Fig. 2. Pore size distribution in 4 samples with 10, 20 and 30 vol. $\%$ of space holders and in three-layer sample.
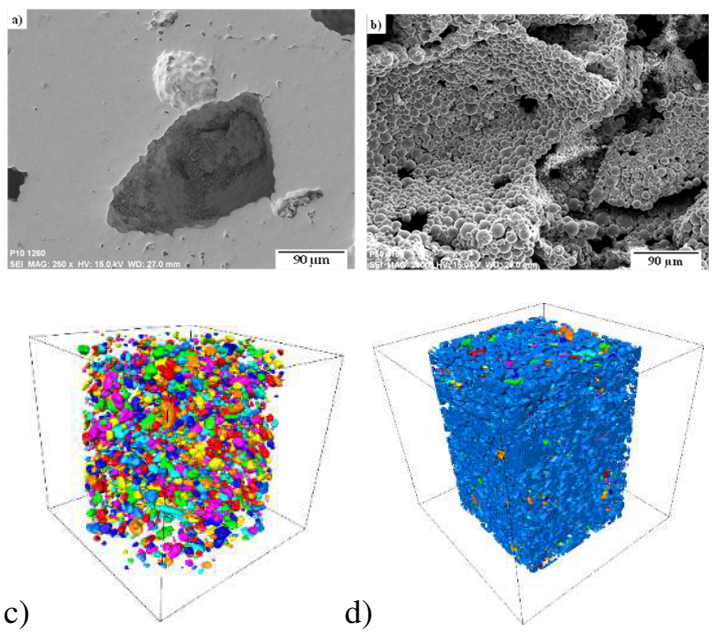

Fig. 3. Microstructure of porous samples, SEM micrographs a) 10 vol. $\%$ of salt and b) 50 vol.\%, 3D rendering of porosity, c) $10 \mathrm{vol} \%$, and d) $30 \mathrm{vol} . \%$.

\subsection{Composite compacts}

Fig. 4 shows SEM micrographies of fractured composite samples. TiN particles were found embedded in the Ti64 matrix as isolated particles (Fig. 4a) or they formed small agglomerates when their amount increased (Fig. 4b). From Fig. 4a it can be observed that pores are nearly spherical and the shape of initial particles is indiscernible when the relative density is close to $90 \%$ for the highest sintering temperature. On the other hand, larger irregular pores are observed when the relative density is lower than $80 \%$ and the spherical shape of Ti-64 particles remains discernible, which indicates a lower densification, for the lowest sintering temperature.
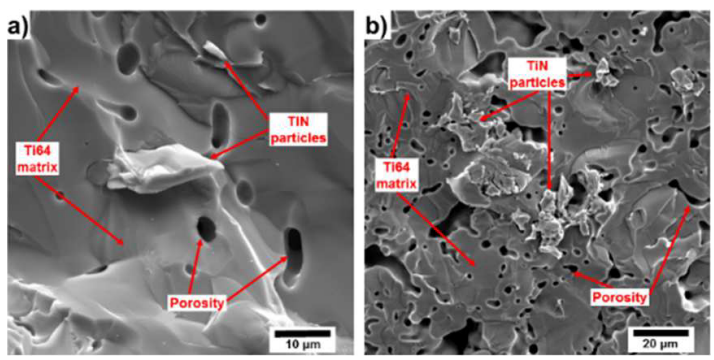

Fig. 4. SEM micrographs of fractured composite samples: a) 5 vol. $\%$ of $\mathrm{TiN}, 1400{ }^{\circ} \mathrm{C}$ and b) 15 vol. $\%, 1200{ }^{\circ} \mathrm{C}$.

Vickers microhardness tests were performed on polished cross-sections of the sintered samples by means of a Future Tech FM-800 microindenter with a load of $500 \mathrm{gf}$ and a dwell time of $20 \mathrm{~s}$. The microhardness of the composite samples is shown in Fig. 5. For each fraction of reinforcement, the microhardness increases with increasing relative density. For a given density it increases with increasing volume fraction of reinforcement particles. With regard to the samples with $89 \%$ relative density, adding only 10 vol.\% of $\mathrm{TiN}$ particles multiplied the microhardness by a factor of 1.8. All reinforced materials exhibit higher hardness than unreinforced ones, although the latter are denser. SEM analysis showed that the addition of TiN particles caused a change in the microstructure of Ti6Al4V matrix from lamellar to equiaxed grains, which is also characterized by a decrement in the amount of $\beta$ phase with increasing temperature. Thus, the rise in microhardness is associated to both the presence of hard inclusions and the refinement of the microstructure within the matrix.

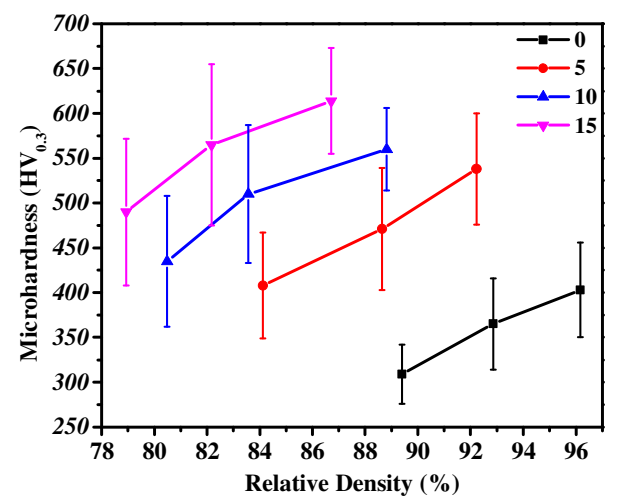

Fig. 5. Microhardness as a function of the relative density for $0,5,10$ and 15 vol.\% of inclusions. 


\subsection{Three-layer sample}

Fig. 6a shows a cross section of the three-layer sample. The three layers, named dense, porous and composite, respectively, are clearly observed from the top to the bottom in the image. Larger pores and reinforcing particles are randomly distributed inside the matrix, which seems to be denser at the interface between the dense and porous layers. Larger interparticle pores are seen at the edges, which is mainly caused by the constrained sintering due to the different densification rates. Fig. $6 \mathrm{~b}$ shows a 3D rendering of the porosity. It is observed that large pores remain connected after sintering. Cracks formed at the interface between the porous and composite layers as well as larger porosities at the interphase between porous and dense layers. Far from the interphases, sintering was normal. Therefore, the porosity was reduced and a disconnected network of pores was obtained.
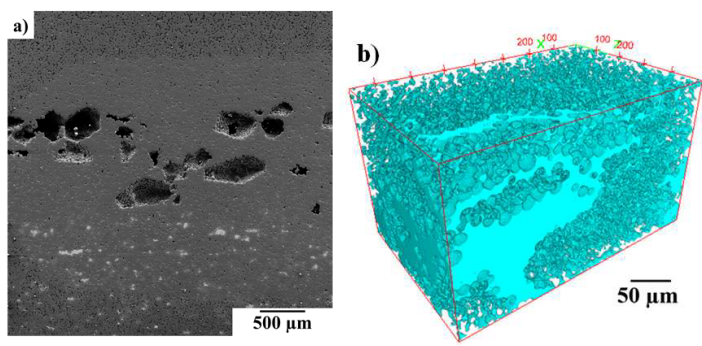

Fig. 6. Microstructure of the three-layer sample, a) SEM cross-section, b) 3D rendering of porosity.

\subsection{Mechanical properties}

After sintering, the bottom and top sample surfaces were polished and compression tests were performed under ASTM D695-02 using an Instron 1150 universal mechanical testing machine at a displacement rate of $0.5 \mathrm{~mm} \cdot \mathrm{min}^{-1}$ [5]. The Young's modulus (E) and the yield strength $\left(\sigma_{y}\right)$ were estimated from the stress-strain curve. It was found that $\mathrm{E}$ and $\sigma_{0.2 \%}$ values increased with increasing relative density in reasonable accordance with different model proposed. Fig. 7 depicts E as a function of $\sigma_{0.2 \%}$ for all samples fabricated in this study. The points show a linear trend, which is surprising since the samples cover a wide range of microstructure. However, it should be noted that the points corresponding to the composite samples lie below the fitted line, indicating that TiN particles increase the yield stress at equivalent Young's modulus.

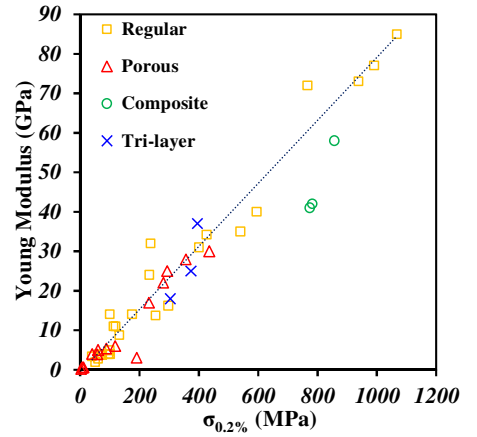

Fig. 7. Young's modulus as a function of yield strength.

\section{Conclusions}

This paper presented a unique set of data on powder processing and characterization of titanium alloy based compacts with complex microstructures. It demonstrated that physical characteristics and mechanical properties of Ti6Al4V alloy can be controlled by the addition of space holders and/or hard inclusions. The fabrication of an original threelayer sample also showed the feasibility of producing components with specific and localized features by traditional powder metallurgy. The mechanical properties obtained for the different materials span across wide ranges of Young's modulus and yield stress, which are consistent with cortical bone properties. This kind of materials could thus be used for fabricating tailored bone implants with microstructure and properties close to those found in real human bones.

\section{Acknowledgments}

The authors wish to thank SEP-CONACYTANUIES, ECOS Nord (Grant 262907) and Universidad Michoacana de San Nicolás de Hidalgo for offering financial support and facilities.

\section{References}

[1] Y. Yan, G.L. Nash, P. Nash, Int. J. Fatigue 55, 81 (2013).

[2] M.M. Dewidar, J.K. Lim, J. Alloy. Compd. 454,442 (2008).

[3] L. Olmos, C.L. Martin, D. Bouvard, D. Bellet, M. Di Michiel, J. Amer. Ceram. Soc. 92, 492 (2009).

[4] H. Galarraga, D.A.Lados, R.R. Dehoff, M.M. Kirka, P. Nandwana, Addit. Manuf. 10, 47 (2016).

[5] M. Guden, E. Celik, E. Akar, S. Cetiner, Mater. Charact. 54(4), 399 (2005). 